\title{
MULTIPLE-SHEETED SPACES AND MANIFOLDS OF STATES OF MOTION*
}

\author{
BY \\ HAROLD HOTELLING
}

Every closed orientable manifold can be represented topologically by a generalized Riemann surface consisting of a number of spaces, each with a single point at infinity, which are considered superimposed and which are joined with each other along certain branch cuts of dimensionality one less than that of the manifold. This has been proved by Professor J. W. Alexander, $\dagger$ who also pointed out that the branch cuts' boundaries may be taken as non-singular non-intersecting manifolds.

But the straightforward reduction of a manifold otherwise defined to such a form is likely to be very tedious and to yield a space having an unnecessarily large number of sheets. Consider for example the threedimensional manifolds of states of motion used by Poincaré and Birkhoff in connection with dynamical problems having two degrees of freedom and studied topologically by the present writer in a paper hereinafter as $A$. In following the proof by Alexander of the existence theorem mentioned, the most obvious way is to represent the manifold by a polyhedron as in $A$, and to divide each face into triangles and the manifold into tetrahedra having a common vertex and these triangles as bases. The vertices of each tetrahedron must all be distinct points. The manifold of states of motion on a surface of genus $p$ is in this way divided into some $24 p-4$ tetrahedra. After fitting together all these cells to form a multiple-sheeted space there will remain the not inconsiderable task of so altering the branch system as to get rid of singularities. An attempt to carry through these operations is enough to show the need of shorter methods in applications.

With the aid of the theorems of $\S \S 1$ to 6 , multiple-sheeted spaces with non-singular branch systems can easily be set up for many three-dimensional manifolds, including manifolds of states of motion, orientable product manifolds, and all manifolds of genus unity. The application to manifolds

\footnotetext{
* Presented to the Society, San Francisco Section, April 4, 1925, and October 31, 1925; received by the editors in July, 1925.

† Bulletin of the American Mathematical Society, vol. 26 (1920), pp. 370-372.

$\ddagger$ Three-dimensional manifolds of states of motion, these T r a n s a t i i n s, vol. 26 (1925), pp. 329-344.
} 
of genus unity is made in $\$ \S 5$ and 6 , to manifolds of states of motion in $\$ \$ 7$ to 10 , and to product manifolds in $\S 11$.

The term "covering space," which has been suggested by Kérékjárto, will be used to denote these generalized Riemann surfaces. It seems a better name than "Riemann space," which is in use in a very different sense.

A covering space representing a manifold is never unique, since the number of sheets can be increased in various ways without altering the topology of the manifold. How far, on the other hand, the number of sheets can be reduced is one of the unanswered questions of analysis situs, except that in the two-dimensional case it is known that the number can always be reduced to two. Whether the covering spaces here found to represent various manifolds are in this sense the simplest possible cannot, therefore, now be known. But it is difficult to see how any further simplification is possible.

1. We shall use special coördinates $\rho, \theta, \varphi$ derived from ordinary cylindrical coördinates $r, \theta, z$, by the equations

$$
\begin{aligned}
r-a & =\rho \cos \varphi, \\
z & =\rho \sin \varphi,
\end{aligned}
$$

where $a$ is a positive number.

The locus for which $\rho=b$, where $b$ is a constant less than $a$, is a tore. A closed sensed curve deformable on this tore into a sensed curve for which $\theta$ is constant and $\varphi$ increases through $2 \pi$ as the curve is traversed in the positive sense will hereafter be referred to as an $\alpha$-curve. It bounds a 2-cell in the interior of the tore. Any closed sensed curve deformable on the tore into a sensed curve for which $\varphi$ is constant and $\theta$ increases through $2 \pi$ as the curve is traversed in the positive sense will be called a $\beta$-curve. A $\beta$-curve evidently bounds a 2 -cell on the exterior of the tore. We shall also call a curve on any surface of genus 1 which bounds a 2 -cell on its interior an $\alpha$-curve, and one which bounds a 2 -cell on its exterior, a $\beta$ curve. A closed curve deformable into one which traverses an $\alpha$-curve $m$ times and a $\beta$-curve $n$ times-operations which by a fundamental property of the tore are commutative-will be called $\alpha^{m} \beta^{n}$.

Consider now a strip generated by the motion of a straight segment which at each stage of the motion lies in a plane $\theta=$ constant, with one end on the curve $\varphi=n \theta, \rho=c$, and the other on the curve $\varphi=n \theta+\pi, \rho=c$, where $c \leqq b$. Such a strip will be said to have an $n$-fold twist. If $n$ is a positive or negative integer or zero the boundaries are distinct curves which are said to have linkage with each other of order $n$. The same terminology will be used for any figure with which this is isotopic. If $n$ is an odd multiple of $\frac{1}{2}$, the strip 
has only one boundary and is one-sided. We shall consider only nonsingular strips, and consequently no other values of $n$ will appear.

Let two sheets cover a space of inversion and join each other along a strip having an $(n / 2)$-fold twist. Let a tore which surrounds the strip in one sheet be deformed continuously until it lies on the strip, covering it doubly; let it then pass into the other sheet and finally cover the initial position. This deformation determines a transformation $\Delta_{n}$ into itself of a tore in the fundamental space which is covered by the initial and final positions of the moving tore.

This transforwation $\Delta_{n}$ is such that an $\alpha$-curve goes into a curve $\alpha^{-1}$ and a $\beta$-curve goes into a curve $\alpha^{n} \beta$; a statement which we may write

or in matrix notation

$$
\begin{aligned}
& \alpha^{\prime}=\alpha^{-1}, \\
& \beta^{\prime}=\alpha^{n} \beta ;
\end{aligned}
$$

$$
\Delta_{n}=\left\|\begin{array}{rr}
-1 & 0 \\
n & 1
\end{array}\right\| \text {. }
$$

To prove this we observe that the deformation may be performed in such a way that each point of the tore in the fundamental space goes into a point which lies in the same half-plane $\theta=$ constant, and is the image of its transform under a reflection on the segment in which this half-plane meets the strip. The point $(\rho, \theta, \varphi)$ then goes into the point $\left(\rho, \theta, \varphi^{\prime}\right)$, where $\varphi^{\prime}+\varphi=n \theta$. Consequently a $\beta$-curve for which $\varphi$ is constant goes into a curve for which $\varphi^{\prime}$ varies through an angle $2 n \pi$ as $\theta$ varies through $2 \pi$, and is therefore a curve of type $\alpha^{n} \beta$. An $\alpha$-curve obviously goes into its inverse.

2. The region between two coaxial tores of which one is within the other is carried by an inversion about a point $P$ of this region, followed by a deformation, into the region exterior to a pair of linking tores. For since each tore is accessible from $P$ before the inversion, each must be accessible from the point at infinity afterwards. Also it is a property of inversions to carry linking circles into linking circles; so that, because certain circles of one tore link certain circles of the other before the inversion, the same must be true afterwards, so that the tores must link.

3. A consequence of this, to be used in $\$ \S 7$ and 8 , is that the region between a tore and $p$ others which are coaxial with and within it, but exterior to each other, is homeomorphic with the region exterior to $p+1$ tores of which one links all the rest but which are not otherwise linking.

4. Another consequence of $\S 2$ is that a tore $T$ may be deformed into a tore $T^{\prime}$ which it links, through a non-singular set of intermediate posi- 
tions which fill the space exterior to the two tores. The transformation $D$ of $T$ into $T^{\prime}$ is such that an $\alpha$-curve and a $\beta$-curve of $T$ go respectively into a $\beta$-curve and an $\alpha$-curve of $T^{\prime}$, provided we define appropriately the senses of these curves. We write

$$
D=\left\|\begin{array}{ll}
0 & 1 \\
1 & 0
\end{array}\right\|
$$

and note in passing that $D^{-1}=D$ and that $\Delta_{n}{ }^{-1}=\Delta_{n}$.

5. A manifold of genus 1 is one whose Heegaard diagram* is a tore. The interior of this tore represents a part of the manifold; on its surface is traced a characteristic curve, which bounds in the other part.

If the characteristic curve is of type $\alpha \beta^{n}$ the manifold is homeomorphic with a two-sheeted covering space whose sheets join along a strip having an $(n / 2)$-fold twist. For let the tore of the diagram link the strip, and let the region be deformed in such a manner that its surface undergoes first the transformation $D$ and then $\Delta_{n}$. Thus $\alpha \beta^{n}$ is transformed first into $\alpha^{n} \beta$ and then into $\beta$, which bounds in the remainder of the manifold, the exterior of the tore in the second sheet.

6. We shall now show that any manifold of genus 1 is represented by a sequence of sheets of which each joins each of its neighbors in the sequence along a twisted strip. In this covering space the sequence of sheets determines a sequence of strips of which each links in the simplest manner each of its neighbors in the sequence. A tore in the first sheet, linking the first strip, may by alternate applications of the deformations of $\S 1$ and $\S 4$ be deformed into a tore in the last sheet which links the last strip. In this process all the intermediate sheets are swept out. If the successive strips have twists of orders $q_{1} / 2, q_{2} / 2, \cdots, q_{p} / 2$, the transformation of the initial position of the tore into the final position is $S=D \Delta_{q_{p}} D \Delta_{q_{p-1}} \ldots$ $D \Delta_{q_{1}} D$.

Let the characteristic curve on the Heegaard diagram be $\alpha^{m} \beta^{n}$. Our proposition will be proved if we can find numbers $q_{1}, \cdots, q_{r}$, positive or negative integers or zero, such that $S\left(\alpha^{m} \beta^{n}\right)=\alpha$ or $\alpha^{-1}$.

The transformation of one into the other of the two surfaces bounding regions each of which represents a part of the manifold may be written

$$
\begin{aligned}
& \alpha^{\prime}=\alpha^{m} \beta^{n}, \\
& \beta^{\prime}=\alpha^{2} \beta^{t},
\end{aligned}
$$

where, since the transformation is reciprocally one-to-one, $m t-n s= \pm 1$.

*Cf. $A, \S 2$. 
Hence $m$ and $n$ are prime to each other. Without loss of generality we shall assume $m$ and $n$ positive and $n>m$.

Now $D \Delta_{q_{1}} D\left(\alpha^{m} \beta^{n}\right)=\alpha^{m} \beta^{q_{1}^{m-n}}$. If we let $q_{1}$ equal the integral quotient obtained in dividing $n$ by $m$, the exponent of $\beta$ becomes the negative of the remainder $r_{1}$ and is therefore numerically less than $m$. If we then apply the transformation $D \Delta_{q_{2}}$ to $\alpha^{m} \beta^{-r_{1}}$, we obtain $\alpha^{-r_{1}} \beta^{-m-r_{1} q_{2}}$. Here let $q_{2}$ be the negative of the quotient obtained in dividing $m$ by $r_{1}$. The exponent of $\beta$ in the last expression is the negative of the remainder, $r_{2}$, and so is less than $r_{1}$. Proceeding in this way we apply successively the transformations $D \Delta_{q_{i}}$, letting $q_{i}$ take the value of the $i$ th quotient obtained in the process of finding the greatest common divisor of $m$ and $n$, multiplied by $(-1)^{i}$. If we denote the successive remainders in this process by $r_{1}, r_{2}$, etc., $\alpha^{m} \beta^{n}$ is transformed successively into

$$
\alpha^{m} \beta^{-r_{1}}, \quad \alpha^{-r_{1} \beta^{-r_{2}}}, \quad \alpha^{-r_{2}} \beta^{r_{3}}, \quad \alpha^{r_{3}} \beta^{r_{4}}, \text { etc., }
$$

the exponents continually decreasing in absolute value. Since $m$ and $n$ are prime to each other, one of the letters $\alpha, \beta$ will eventually take 1 or -1 as its exponent; the other exponent will then become zero. If $\alpha^{m} \beta^{n}$ is in this way transformed into $\beta^{ \pm 1}$, a further transformation $D \Delta_{0}$ will carry it into $\alpha^{ \pm 1}$. Having determined the values of $q_{1}, \cdots, q_{p}$ which accomplish this we have proved the proposition.

Use has been made of the fact that $\alpha$ and $\beta$ are commutative. The absence of this property for curves on surfaces of genus greater than one prevents an immediate generalization of the method.

\section{MANIFOLDS OF STATES OF MOTION}

7. The motion of a particle on a surface at any instant is specified by three parameters, two giving the position on the surface and one the direction; the energy is assumed constant. These three parameters also specify a point in a manifold whose topological properties depend only on those of the portion of the characteristic surface on which the particle's total energy is positive. Covering spaces representing these manifolds have been found in $A$ for the cases in which the connectivity of the surface does not exceed that of the tore, and also for all cases in which there are boundaries. With the help of $\$ \S 1$ to 4 we now find covering spaces representing manifolds of states of motion on the remaining surfaces.

Divide an orientable surface of genus $p$ into two parts by $p+1$ nonintersecting non-homologous curves and map each part conformally on a plane region within a circle and outside of $p$ smaller circles. The states of motion on one of these parts are represented by the points of a region $R$ 
between a tore and $p$ smaller tores within it and having the same axis with it, but exterior to each other. Translation of a vector around one of the boundaries of the plane map is represented by an $\alpha$-curve on one of the tores; while rotation of a vector at a point through an angle $2 \pi$ is represented by a $\beta$-curve if opposite directions on the surface are treated as distinct, by a $\beta^{2}$-curve if opposite directions are considered identical.

This region $R$ is, according to $\S 3$, homeomorphic with the part of a space of inversion $S$ exterior to $p+1$ tores $T_{0}, T_{1}, \cdots, T_{p}$ of which one, $T_{0}$, links all the rest. The homeomorphism carries $\alpha$-curves into $\beta$-curves and $\beta$-curves into $\alpha$-curves on $T_{0}$, but does not change the classes of curves on $T_{1}, \cdots, T_{p}$.

The states of motion on the other part of the surface are likewise represented by the points of a region $R^{\prime}$ within a tore and outside of $p$ others; and $R^{\prime}$ is homeomorphic with a region in a sheet $S^{\prime}$ covering that in $S$ which corresponds to $R$.

To see how the tores bounding $R$ are matched with those bounding $R^{\prime}$ we find by the method of $A, \S 3$, that translation of a vector around a boundary of one of the plane maps is equivalent to motion, with rotation through an angle $4 \pi$, around the corresponding boundary of the other map. This presupposes such orientations of the maps that a positive rotation on a boundary of one is equivalent to a negative rotation on the other. Hence the transformation of a boundary of $R$ into a boundary of $R^{\prime}$ is

$$
\alpha^{\prime}=\alpha \beta^{2 \epsilon}, \quad \beta^{\prime}=\beta^{-1},
$$

where $\epsilon=1$ if opposite directions are considered distinct and $\epsilon=2$ if opposite directions are considered identical. Of the boundaries in $S, T_{1}, \cdots, T_{p}$ will be transformed respectively into boundaries $T_{1}{ }^{\prime}, \cdots, T_{p}{ }^{\prime}$ of $S^{\prime}$ in such a way that (1) holds; while for the exceptional tores $T_{0}$ and $T_{0}{ }^{\prime}$ which link the others the transformation is expressed by interchanging $\alpha$ with $\beta$ and $\alpha^{\prime}$ with $\beta^{\prime}$ in (1), so that

$$
\alpha^{\prime}=\alpha^{-1}, \quad \beta^{\prime}=\alpha^{2 \epsilon} \beta .
$$

But (2) is precisely the transformation $\Delta_{n}$ of $\S 1$, with $n=2 \epsilon$. We therefore join the sheets $S$ and $S^{\prime}$ along a strip which has an $\epsilon$-fold twist, its boundaries being curves of type $\alpha^{\epsilon} \beta$ on the tores $T_{0}$ and $T_{0}^{\prime}$.

The tores $T_{1}, \cdots, T_{p}$ bounding the region in $S$ are deformed into the respectively corresponding tores $T_{1}{ }^{\prime}, \ldots, T_{p}{ }^{\prime}$ in $S^{\prime}$ after interposing two new sheets $S_{i}$ and $S_{i}{ }^{\prime}$ for each $T_{i}$. The transformation (1) of $T_{i}$ into $T_{i}{ }^{\prime}$ is equivalent to $\Delta_{0} D \Delta_{-2 \epsilon} D \Delta_{0}$. A deformation of this type is possible which sweeps out $S_{i}$ and $S_{i}{ }^{\prime}$ if $S$ and $S_{i}$ join along a strip having a 0 -fold twist, 
$S_{i}$ and $S_{i}{ }^{\prime}$ along a strip having a $(-\epsilon)$-fold twist, and $S_{i}{ }^{\prime}$ and $S^{\prime}$ along a strip having a 0 -fold twist.

We thus have $2 p+2$ sheets. A picture of the complete set of branch curves is obtained, for $p=2$ and $\epsilon=1$, by deleting from the figure on page 489 the four curves at the top.

8. States of motion on one-sided surfaces. A non-orientable surface of odd connectivity can, like an orientable surface, be represented by a pair of plane regions, each bounded by circles, with the one difference that some of the circles of one map are to be identified with those of the other in a sense-preserving and some in a sense-reversing manner, whereas in the orientable case the mode of identification is the same for all the circles. If the connectivity of the surface is $2 p+1$, the boundary of each map will consist of $p+1$ circles of which the number in each of the two classes is at least one but is otherwise arbitrary.

Thus the manifold of states of motion consists of two regions $R$ and $R^{\prime}$ of which some of the boundaries match as in the last section and some in a different manner. This matching is determined by a geometrical argument similar to that of $A, \S 3$. It may be shown that if a circle of one map matches a circle of the other map in a sense-reversing manner, a vector at a point $\theta$ of one circle making an angle $\varphi$ with a fixed direction in the plane is to be identified with a vector at a point $\theta^{\prime}$ of the other circle making an angle $\varphi^{\prime}$ with this fixed direction, where

$$
\begin{aligned}
\theta^{\prime} & =-\theta, \\
\varphi^{\prime} & =\varphi-2 \theta,
\end{aligned}
$$

with an arbitrary constant added in each case. This shows that the transformation connecting the corresponding boundaries of $R$ and $R^{\prime}$ is of the class

$$
\alpha^{\prime}=\alpha^{-1} \beta^{-2 \epsilon}, \quad \beta^{\prime}=\beta ;
$$

for variation of $\theta$ through $2 \pi$ with $\varphi$ constant will, according to (3), cause $\theta^{\prime}$ to vary through $-2 \pi$ and $\varphi^{\prime}$ through $-4 \pi$; while variation of $\varphi$ with $\theta$ constant causes $\varphi^{\prime}$ to vary through an equal angle.

Mapping $R$ and $R^{\prime}$ respectively upon $S$ and $S^{\prime}$ as before, we see that each of the tores $T_{1}, \cdots, T_{p}$ is to be deformed into one of the tores $T_{1}{ }^{\prime}, \cdots, T_{p}{ }^{\prime}$ in such a way that either (1) or (4) holds; while for $T_{0}$ and $T_{0}{ }^{\prime}$ the transformation is either (2) or that derived from (4) in the same way that (2) is derived from (1); namely

$$
\alpha^{\prime}=\alpha, \quad \beta^{\prime}=\alpha^{-2 \epsilon} \beta^{-1} .
$$


Any of these transformations may be effected by deformation through intermediate sheets; for (4) is equivalent to $\Delta_{0} D \Delta_{0} D \Delta_{0} D \Delta_{-2 \epsilon} D \Delta_{0}$, and (5) to $\Delta_{0} D \Delta_{0} D \Delta_{2 \epsilon}$, while it has been seen in $\S 7$ that (1) is equivalent to $\Delta_{0} D \Delta_{-2 \epsilon} D \Delta_{0}$ and (2) to $\Delta_{2 \epsilon}$. There is also an infinite number of other modes of expressing these transformations in terms of sequences of $D$ 's and $\Delta$ 's alternating, with a $\Delta$ on each end. For example, any of these transformations may be followed by any number of transformations such as $\Delta_{n} D \Delta_{0} D \Delta_{-n} D \Delta_{0} D$, which is equivalent to the identity.

The simplest of the corresponding covering spaces has $2 p+4$ sheets $S, S^{\prime}, S_{0}, S_{1}, \cdots, S_{p}, S_{0}{ }^{\prime}, S_{1}{ }^{\prime}, \cdots, S_{p}{ }^{\prime}$, of which $S$ is joined to $S_{0}, S_{1}, \cdots, S_{p}$ and $S^{\prime}$ to $S_{1}{ }^{\prime}, \cdots, S_{p}{ }^{\prime}$ along strips of zero twist; $S_{0}{ }^{\prime}$ is joined to $S_{0}$ along a strip of zero twist and to $S^{\prime}$ along a strip of $\epsilon$-fold twist; while $S_{i}(i=1, \cdots, p)$ is joined to $S_{i}{ }^{\prime}$ along a strip of $(-\epsilon)$-fold twist.

9. Surfaces of even connectivity; $\epsilon=1$. A surface of even connectivity, necessarily one-sided, is defined* as the sum, modulo 2 , of an orientable surface and a projective plane having a 2-cell in common. We may therefore expect to find a covering space representing the states of motion on such a surface by a suitable combination of that of $\S 7$ with a covering space representing the states of motion on a projective plane. A space of the latter kind was found in $A, \S \S 5-8$, for the case in which opposite directions are considered distinct, a case which we shall now discuss. This covering space has two sheets which join along a strip having a 2 -fold twist (illustrated in $A$, Fig. 3).

Let the 2-cell common to the orientable surface and the projective plane be $J$ and let its boundary be $j$. The removal of $J$ from either surface implies the removal from the manifold of states of motion of the interior or exterior of a tore $T$ which represents the states of motion on $j$. In the manifold of $\S 7$ we shall let $T$ be a tore in the sheet $S$ which links the strip joining $S$ and $S^{\prime}$. It will then be the interior of $T$ that represents states of motion on $J$. Translation along $j$ will be represented by an $\alpha$-curve of $T$, and rotation at a point by a $\beta$-curve. Translation is of course defined with reference to a system of curves on $J$ which is the image of a system of parallels in a plane.

In order to combine the two covering spaces we must find in that for the projective plane a region, homeomorphic with the interior of a tore, which represents the states of motion on the 2-cell $J$. We must also determine what curves on the boundary of this region represent translation of a vector along $j$, and what curves represent rotation at a point. A curve

* O. Veblen, Cambridge Colloquium Lectures, p. 48. 
of the first kind can, by taking $J$ sufficiently small, be enclosed by as small a sphere as we please, for the variation in direction of the vector is zero. Such a curve can therefore be deformed to a point in the manifold, and may be taken at will as a curve $\alpha$ or $\alpha^{-1}$ on the tore.

Rotation of a vector at a point of $j$ will be represented on the tore by some curve of type $\alpha^{n} \beta^{ \pm 1}$. We may determine $n$, as well as the manner of the tore's winding about, by finding how to mark into Fig. 3 of $A$ two curves of which each represents rotation at a point, for these curves will have with each other linkage of order $n$.

For this purpose we examine a correspondence of the two-sheeted space with the cylindrical region of Fig. 4 of $A$. A plane $s$ parallel to the bases of this cylinder and half way between them divides it into two regions $R_{1}$ and $R_{2}$. The points of the curved surface are to be identified in pairs as specified in $A, \S 5$. If this identification be made only for the point pairs of which both points lie on the surface of $R_{1}$, this region becomes homeomorphic with the interior of a tore; and similarly for $R_{2}$. The regions $R_{1}$ and $R_{2}$ are then canonical regions (cf. $A, \S 2$ ). The canonical cuts are represented on the cylindrical surface by four 2-cells, each of which is bounded by an arc on $s$, an arc on a base of the cylinder, an arc of one of the helices shown in Fig. 4 of $A$, and an arc of another helix congruent with this one.

It is not difficult to show that a correspondence $F$ between cylindrical region and covering space may be set up under which each of the regions $R_{1}$ and $R_{2}$ corresponds in 1-1 continuous fashion to one sheet and under which one of the branch curves of Fig. 3, $A$, which we shall call $\gamma$, is the correspondent of the two generators of the cylinder shown in Fig. 4, $A$; these we shall call $\gamma^{\prime}$ and $\bar{\gamma}^{\prime}$. The double strip bounded by the branch curves, along which the sheets join, is of course the correspondent under $F$ of the common surface of $R_{1}$ and $R_{2}$.

Regarding Fig. 3, $A$, as a projection of the branch curves on a plane, we may consider that the four narrow regions in that figure, each bounded by two arcs, represent the strip bounded by the branch curves, while the central four-sided region represents the two canonical cuts, one in each sheet.

Let $\gamma_{1}{ }^{\prime}$ and $\gamma_{2}{ }^{\prime}$ be two lines within the cylinder parallel to $\gamma^{\prime}$, and let $\gamma_{1}$ and $\gamma_{2}$ be curves in the covering space which are the respective correspondents under $F$ of $\gamma_{1}{ }^{\prime}$ and $\gamma_{2}{ }^{\prime}$. Each of the curves $\gamma^{\prime}, \gamma_{1}{ }^{\prime}, \gamma_{2}{ }^{\prime}$, and therefore $\gamma, \gamma_{1}, \gamma_{2}$, represents rotation of a vector at a point. But while $\gamma^{\prime}$ and $\bar{\gamma}^{\prime}$ lie entirely on the common surface of $R_{1}$ and $R_{2}, \gamma_{1}{ }^{\prime}$ and $\gamma_{2}{ }^{\prime}$ consist each of two segments, one in $R_{1}$ and one in $R_{2}$. 
Since $\gamma_{1}{ }^{\prime}$ has no point in common with either canonical cut, $\gamma_{1}$ may be projected upon Fig. 3, $A$, in such a way that the projected curve has no point within the central four-sided region. Therefore, since $\gamma_{1}$ cuts only twice the strip bounded by $\gamma$ and the other branch curve, and since $\gamma_{1}$ may be taken arbitrarily close to $\gamma$, it cannot link $\gamma$.

In the same way it may be shown that $\gamma_{2}$ does not link $\gamma$. From a consideration of the relations of $\gamma_{1}{ }^{\prime}, \gamma_{2}{ }^{\prime}$ and $\gamma^{\prime}$ it will be seen further that $\gamma_{1}$ is deformable to $\gamma$ through a set of intermediate positions containing no point of $\gamma_{2}$. Hence $\gamma_{1}$ does not link $\gamma_{2}$.

Thus $n=0$, and rotation of a vector at a point of $j$ is represented by a $\beta$-curve on the tore in the two-sheeted covering space whose interior represents states of motion on $J$. The matching of this tore with the tore $T$, of the covering space representing states of motion on the orientable surface, is determined topologically simply by

$$
\alpha^{\prime}=\alpha^{ \pm 1}, \quad \beta^{\prime}=\beta^{ \pm 1},
$$

where we may take the signs as we please. Hence the two spaces are to join along a strip of zero twist bounded by curves such as $\gamma_{1}$ and $\gamma_{2}$. Denote the sheets of the two-sheeted space by $\bar{S}$ and $\bar{S}^{\prime}$. Each of these sheets will join $S$ along a part of the strip just mentioned, for it pierces twice the strip along which $\bar{S}$ and $\bar{S}^{\prime}$ join each other.

The system of branch curves for a covering space representing states of motion on a surface of connectivity 6 is shown in the figure on page 489 .

10. The manifolds of states of motion on surfaces of even connectivity with opposite directions treated as identical are more complicated and can apparently be represented as covering spaces only by a rather laborious process. As it is not clear that much can be gained in applications by requiring opposite directions to be identified, we shall give this case very summary treatment.

The manifold of states of motion on a projective plane is in this case represented by a cylindrical region of height $\pi$. The two bases are to be identified in the manner determined by a translation, and the point of the curved surface whose cylindrical coördinates (the axis of the coördinates being the axis of the cylinder) are $(r, \theta, \varphi)$ is to be identified with the point $(r, \theta+\pi, 2 \theta-\varphi)$, the last coördinate being reduced modulo $\pi$. (In the case of distinct opposite directions the reduction was modulo $2 \pi$.) A plane $s$ parallel to the bases of the cylinder and half way between them divides the interior into two regions. Two 2-cells on the boundary of each of these regions are to be identified respectively in the manner just indicated with two other 2-cells on the boundary of the same region. In this way a Heegaard diagram of genus 2 is obtained. 


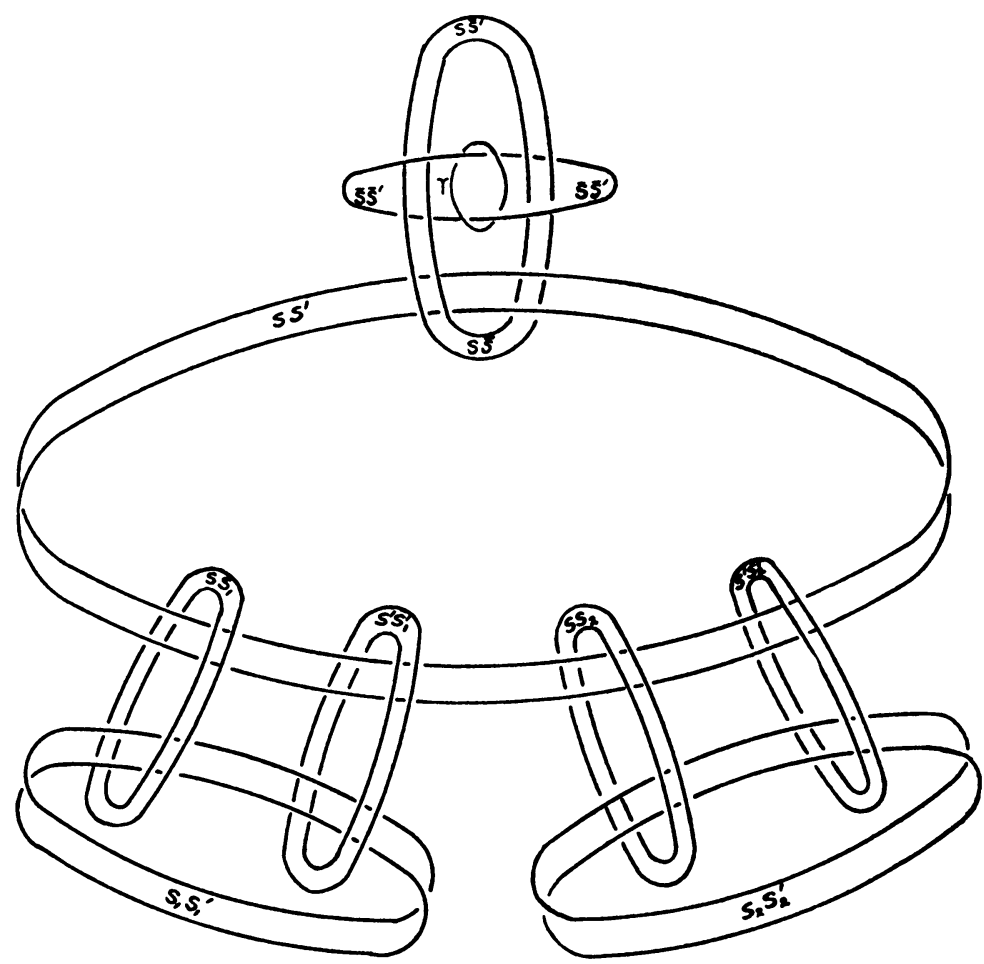

The same Heegaard diagram may be obtained by cutting apart the two sheets of a covering space whose branch curves are three circles, each linking each of the others. Thus the two manifolds may be shown to be homeomorphic.

By tracing in this two-sheeted space a pair of curves, each representing the states of motion at a point, it would be possible in the light of $\S 9$, and with the help of $\$ \S 1$ to 4 , to determine how it should be joined to an appropriate one of the manifolds of $\S 7(\epsilon=2)$ to give a covering space representing states of motion on any surface of even connectivity.

\section{Product MANIFolds}

11. The "product manifolds" whose points are in 1-1 continuous correspondence with pairs of points, one in each of two manifolds called the factors, have been discussed by E. Steinitz* and H. Künneth. $\dagger$

* Beitragen zur Analysis Situs, Sitzungsberichte der Berliner Mathematischen Gesellschaf t, vol. 7 (1908), p. 29.

†Über die Bettischen Zahlen einer Produktmannigfaltigkeit, Mathematische Annalen, vol. 90 (1923), p. 65. 
It is noteworthy that a manifold of the states of motion in an $n$-dimensional manifold, while not in general a product manifold itself, may be divided into regions of which each is the product of an $n$-cell by an $(n-1)$ dimensional sphere (if opposite directions are considered distinct) or projective space (if opposite directions are treated as identical).

The only closed product manifolds which are three-dimensional covering spaces have as factors a closed curve and an orientable surface; for the product of a curve and a one-sided surface is one-sided; while a covering space is necessarily orientable, because a curve in it along which the indicatrix were reversed would have to cover such a curve in the orientable fundamental space.

As in $\$ 7$ let an orientable surface of genus $p$ be mapped on two plane regions, each bounded by $p+1$ circles. The product of each region by a closed curve is, as in $\S 7$, homeomorphic with the space exterior to $p+1$ tores of which one links all the rest. But the manner of joining the sheets is simpler in the present case; for we may set up the homeomorphism in such a way that an $\alpha$-curve on each tore in the first sheet is to be identified with an $\alpha^{-1}$-curve on the corresponding tore in the second sheet, and a $\beta$-curve with a $\beta$-curve. From $\S 1$ it then follows that the manifold is homeomorphic with a two-sheeted space having $2 p+2$ knotless branch curves of which two link all the rest in the simplest fashion, but which do not link otherwise.

Stanford University, Calm. 